\title{
Resveratrol interferes with N-nitrosodiethylamine-induced hepatocellular carcinoma at early and advanced stages in male Wistar rats
}

\author{
DEVARAJA RAJASEKARAN, JAYASUDHA ELAVARASAN, MURUGAN SIVALINGAM, \\ EKAMBARAM GANAPATHY, AKHILANDEESWARI KUMAR, K. KALPANA and DHANAPAL SAKTHISEKARAN \\ Department of Medical Biochemistry, Dr. ALM PG Institute of Basic Medical Sciences, \\ University of Madras, Taramani, Chennai 600113, Tamilnadu, India
}

Received April 4, 2011; Accepted July 21, 2011

DOI: $10.3892 / \mathrm{mmr} .2011 .555$

\begin{abstract}
Resveratrol, a phytochemical compound abundant in red wine and grapes, is known to affect cancer cells both in vitro and in vivo. A great amount of data have indicated the therapeutic benefits of resveratrol against cancer. However, it remains unclear whether these benefits are similar and equally effective in both the early and advanced stages of cancer or carcinogenesis. In this study, we report the effects of resveratrol in the early and advanced stages of hepatocarcinogenesis in a model of N-nitrosodiethylamine (DEN)-induced hepatocellular carcinoma (HCC) of male Wistar rats. For the experiment, rats were divided into different groups and treated with resveratrol either from day 1 of DEN administration for 15 days (preHCC), or after the development of HCC, i.e., 15-16 weeks after DEN administration (post-HCC), and compared to untreated HCC-bearing rats. Biochemical analysis of $\alpha$-fetoprotein, the known serum marker for HCC, and other serum and liver marker enzymes also demonstrated a decreased level upon resveratrol treatment compared to the untreated HCC-bearing rats. H\&E staining of tissue sections from the liver showed alteration or transformation of liver parenchymatous tissue in DEN-induced HCC (at 15-16 weeks). Resveratrol treatment during early (on day 1 of DEN-induction) and advanced (weeks 17-18) HCC showed a marked difference in the tissue architecture compared to untreated HCC. Immunoblot analysis revealed that resveratrol intervention at both the early and advanced stages of DEN-induced HCC activated the apoptotic markers, such as PARP cleavage, caspase-3 activation, p53 up-regulation and cytochrome-c release. In addition, semiquantitative RT-PCR and immunoblot analysis demonstrated the up- and downregulation of key apoptotic regulators, such as $\mathrm{Bax}$ and $\mathrm{Bcl}_{2}$,
\end{abstract}

Correspondence to: Professor Dhanapal Sakthisekaran, Department of Medical Biochemistry, Dr. ALM PG Institute of Basic Medical Sciences, University of Madras, Taramani, Chennai 600113, Tamilnadu, India

E-mail: rdraj79@yahoo.co.in

Key words: hepatocellular carcinoma, resveratrol, apoptosis respectively, in a resveratrol treatment-dependent manner. Our results indicate that the administration of resveratrol either at the early or advanced stages of hepatocarcinogenesis is equally effective and involves the activation of the apoptotic pathway in male Wistar rats.

\section{Introduction}

Cancer incidence and its related death are increasing worldwide. Among solid tumors, primary liver cancer, known as hepatocellular carcinoma (HCC), is the fifth most common malignancy and the third leading cause of cancer-related deaths (1). Surgery, including transplantation, is currently considered the most effective treatment for HCC. However, the majority of patients show poor prognosis and frequent recurrence (2). Earlier reports have revealed that in $>70 \%$ of $\mathrm{HCC}$ patients, the tumor recurrence is inevitable after surgical treatment. Hence, the need to find an alternate therapy is indispensable and has been the main focus of recent research on HCC treatment. Other therapeutic options, such as percutaneous ethanol injection, radiofrequency ablation, intra-arterial therapy and chemotherapy, provide only transient relief, but not a complete cure of the disease nor do they prevent recurrence $(2,3)$. Since the early detection of HCC is difficult due to the lack of specific diagnostic markers and asymptomatic nature of the tumor, patients are always presented with the advanced stage of HCC. Recent developments in chemotherapeutic agents, such as sorafenib, have shown promising results in transiently reducing the tumor burden (4). However, chemotherapeutic agents, in general, are not cancer-specific in targeting and often exhibit a broad spectrum of toxicities (systemic and/or neuronal). Hence, there is a necessity to find a better treatment option/agent that selectively targets and blocks the tumorspecific pathways in HCC.

Among different classes of anticancer agents, plant derivatives, particularly the dietary polyphenols, have received greater attention due to their abundance in natural foods and minimal or no adverse effects. One such polyphenol, resveratrol, chemically known as trans-3,4,5-trihydroxystilbene, is abundant in grapes, berries and peanuts (5). In the past decade, a number of studies have documented the medicinal 
properties and health benefits of resveratrol in cardiovascular protection, neuronal protection and anti-inflammation (6). Ever since Jang et al (7) reported the role of resveratrol against cancer, many studies have demonstrated the anti-proliferative and pro-apoptotic effects of resveratrol in in vitro as well as in vivo tumor models $(8,9)$. Resveratrol has been shown to be effective against several cancer cell lines, such as colon adenocarcinoma (Caco-2), medulloblastoma, metastatic breast cancer (MDA-MB-231) and pancreatic carcinoma cells (10).

Certain pre-clinical studies on HCC have demonstrated the anticancer efficacy of resveratrol both in chemoprevention and chemotherapy $(11,12)$. However, to date, there are no reports on comparing the effect of resveratrol in early and advanced stages of N-nitrosodiethylamine (DEN)-induced hepatocarcinogenesis. Therefore, we investigated the effects of resveratrol on DEN-induced HCC at early as well as advanced stages of hepatocarcinogenesis. In this study, we demonstrate that resveratrol treatment affects the progression of DEN-induced HCC, irrespective of the stages (early or advanced) of hepatocarcinogenesis. Notably, resveratrol treatment activated the apoptotic pathway in both early- and advanced-stage interventions, and the mechanism involved PARP cleavage, caspase- 3 activation, up-regulation of p53 and Bax, down-regulation of $\mathrm{Bcl}_{2}$ and cytochrome-c release. Thus, the activation of a common apoptotic signaling pathway is the principal mechanism underlying resveratrol-mediated anticancer effects, in both early- and advanced-stage therapeutic interventions.

\section{Materials and methods}

Chemicals and reagents. Resveratrol, DEN and bovine serum albumin (BSA) were obtained from Sigma Chemical Co. (St. Louis, MO, USA). Other reagents included polymerase chain reaction reagents (Kapa Biosystem Inc., Woburn, MA, USA), primer sequences (Sigma Chemical Co.), the cDNA synthesis kit, TRIzol, RNase-out (Invitrogen Corp. Carlsbad, CA, USA) and the superscript reverse transcription kit (Invitrogen Corp.). Unless otherwise mentioned, all antibodies, such as anti-caspase-3, poly ADP ribosyl polymerase (PARP), cytochrome-C, $\mathrm{Bcl}_{2}, \mathrm{Bax}, \beta$-actin and $\mathrm{p} 53$, were purchased from Santa Cruz Biotechnology (Santa Cruz, CA, USA). Electrophoretic reagents, such as agarose, acrylamide and N, N'-methylene bisacrylamide, were purchased from GE Healthcare Europe GmbH (München, Germany). All other chemicals used (analytical grade) were obtained from Sisco Research Laboratories Pvt., Ltd. (Mumbai, India) and Glaxo Laboratories (CDH Division, Mumbai, India).

In vivo studies. Healthy male Wistar albino rats (6-8 weeks old) were procured from the Central Animal House Facility, Dr. ALM PG IBMS, University of Madras, Taramani, Chennai, India, and maintained in a controlled environmental condition with a temperature of $23 \pm 2^{\circ} \mathrm{C}$ and a relative humidity of $50-70 \%$ on alternative 12 -h light/dark cycles. All animals were fed a standard pellet diet (Gold Mohor rat feed; M/s. Hindustan Lever Ltd., Mumbai, India) and water ad libitum. This study on male Wistar albino rats was performed according to the guidelines of the Institutional Animal Ethics Committee (IAEC No. 02/073/06) of the University of Madras, Chennai, India.
The experimental animals were randomized into five groups of 6 rats each (Fig. 1). The first group of rats was treated orally with $0.5 \%$ aqueous solution of carboxymethyl cellulose as the vehicle (referred hereafter as 'vehicle control'). The second group of rats was treated with resveratrol (referred as 'Rsv control'). These two non-tumor groups served as the controls for the vehicle as well as the therapeutic test compound (resveratrol), and they were not subjected to DEN-induced hepatocarcinogenesis. The third group of rats was treated with DEN for the induction of HCC. DEN was administered by intra-peritoneal injection $(200 \mathrm{mg} / \mathrm{kg}$ body weight, single dose). This was followed by providing $0.05 \%$ phenobarbital in drinking water after the second week of DEN treatment up to the sixteenth week. The fourth group of rats was subjected to DEN-induced HCC and resveratrol treatment $(20 \mathrm{mg} / \mathrm{kg}$ body weight) simultaneously on day 1 and continued for 15 days. This group was used for the study of the effects of resveratrol during the early stages of DEN-induced HCC, as resveratrol was administered even prior to the complete induction of $\mathrm{HCC}$, and is referred to as 'DEN + Rsv (pre)'. Finally, the fifth group of rats was subjected to HCC induction followed by treatment with resveratrol $(20 \mathrm{mg} / \mathrm{kg}$ body weight, dissolved in $0.5 \%$ aqueous solution of carboxymethyl cellulose, orally) from the 17th to the 18th week of HCC induction (for 15 days). This group was used for studying the therapeutic effects of resveratrol and referred as post-tumor treatment ['DEN + Rsv (post)']. After the completion of the experimental period (18 weeks), the animals were sacrificed and different organs were harvested for further analysis according to the parameters of interest.

For histology, tissues were routinely fixed in phosphatebuffered $10 \%$ formalin (Polysciences Co., Warrington, PA, USA), dehydrated by graded ethanol, embedded in Paraplast Plus wax (McCormick Scientific, IL, USA), sectioned at 5 microns, mounted on slides, oven-dried and deparaffinized. The tissue sections were subjected to H\&E staining and viewed under a light microscope.

Analysis of serum and liver markers. The $\alpha$-fetoprotein (AFP) level was quantified based on the ELISA principle using the Fully Automated Bayer ADVIA Centaur chemiluminescent system (Bayer Corp., Pittsburgh, PA, USA). In brief, a 96-well plate pre-coated with AFP antibody was incubated with an anti-AFP antibody conjugated with horse-radish peroxidase to measure signal intensities generated from the enzymesubstrate reactions. Serum and liver marker enzymes, such as aspartate aminotransferase (AST), alanine aminotransferase (ALT), alkaline phosphatase (ALP), acid phosphatase (ACP) and lactate dehydrogenase (LDH), were assayed as described by King (13). The enzyme assays for 5'-nucleotidase (5'-Nuc) and $\gamma$-glutamyl transpeptidase $(\gamma-\mathrm{GT})$ were performed following the protocols described earlier (14). Protein content was estimated following the method of Lowry et al (15).

Semi-quantitative reverse transcription $(R T)$-polymerase chain reaction $(P C R)$. RNA extraction was performed using TRIzol reagent and the concentration and quality of the isolated RNA was confirmed by standard spectrophotometric analysis. Total RNA $(1 \mu \mathrm{g})$ was subjected to a total volume of $20 \mu \mathrm{l}$ RT-PCR reaction containing $5 \mu \mathrm{l}$ of $10 \mathrm{X}$ RT-buffer, $10 \mathrm{mM}$ 


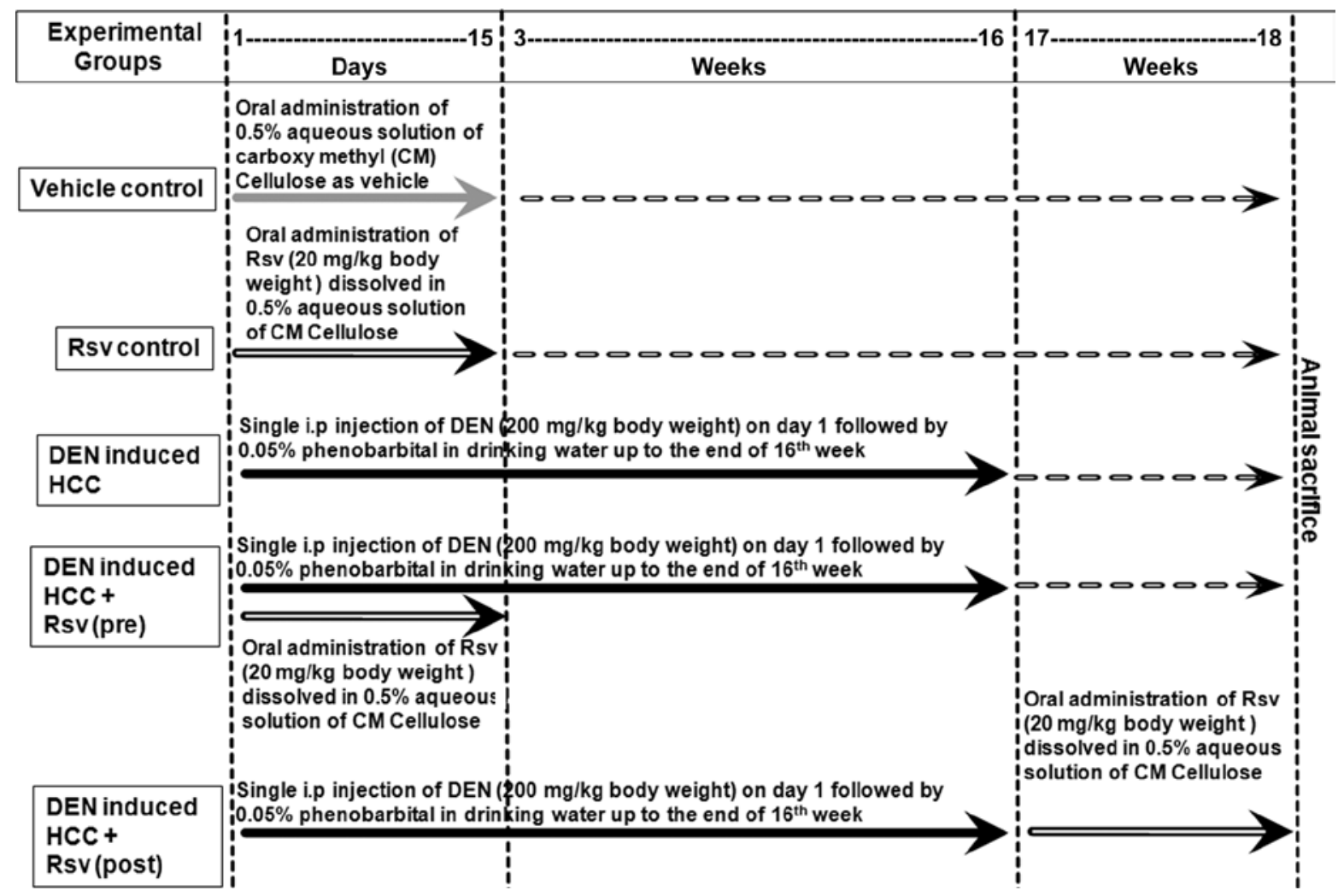

Figure 1. Schematic representation of the experimental design. Each group contained 6 rats. Rsv, resveratrol; DEN + Rsv (pre), DEN-induction of HCC and simultaneous administration of Rsv (from 1 to 15 days); DEN + Rsv (post), DEN-induction of HCC followed by Rsv treatment (from 17 to 18 weeks); i.p., intra-peritoneal. Broken arrow indicates maintenance of animals until sacrifice.

dNTPs, cDNA synthesis mix [50 $\mu \mathrm{M}$ of Oligo(dT)20, $25 \mathrm{mM}$ $\mathrm{MgCl}_{2}, 1 \mu \mathrm{l}$ RNase out (40 U/ $\left.\mu \mathrm{l}\right)$ and $1 \mu \mathrm{l}$ SuperScript-RT]. cDNA synthesis was carried out following the protocol of the manufacturer (Invitrogen Corp.). The cDNA synthesized was subjected to PCR for further analysis of various gene expressions. After amplification, the RT-PCR products were electrophoresed on $2 \%$ agarose gels and stained with ethidium bromide. Images were captured with the Molecular Imager gel documentation unit (Bio-Rad Laboratories, Hercules, CA, USA) and subsequently subjected to densitometry analysis. $G A P D H$ was used as the internal control for normalization and to provide a semi-quantitative measure of the relative changes in gene expression. The primer sequences used for the expression analysis of the respective genes are provided in Table I.

Immunoblotting. Immunoblot analysis was performed as per standard procedure using specific antibodies. Briefly, liver tissue homogenates were prepared in ice-cold Tris-HCl buffer (0.1 M, pH 7.4) from various animal groups subjected to HCC induction and resveratrol treatment. The protein denaturation was achieved by heating at $95^{\circ} \mathrm{C}$ for $10 \mathrm{~min}$ in the presence of SDS and a reducing agent (dithiothreitol or $\beta$-mercaptoethanol). The samples were then subjected to sodium dodecyl sulphate polyacrylamide gel electrophoresis (SDS-PAGE) as previously described (16). The protein profiles were electroblotted onto polyvinylidene difluoride (PVDF) membranes (Millipore Inc., Billerica, MA, USA) at $4^{\circ} \mathrm{C}$ overnight. The antibody incubation procedures and the blocking buffer used were as per the antibody supplier's instructions. The membrane was
Table I. Primer sequences used for semi-quantitative RT-PCR.

Symbol Gene sequence

\section{p53}

Forward

5' GTCGGCTCCGACTATACCACTATC 3'

Reverse

5' CTCTCTTTGCACTCCCTGGGG 3

$\mathrm{Bcl}_{2}$

Forward 5' GGGATGCCTTTGTGGAACTA 3'

Reverse 5' CTCACTTGTGGCCCAGGTAT 3'

Bax

Forward 5' ATGGAGCTGCAGAGGATGAT 3'

Reverse 5' CCAGTTGAAGTTGCCATCAG 3'

GAPDH

Forward

5' GCATGGCCTTCCGTGTTCC 3'

Reverse

then developed using the sensitive chemiluminescence method (Millipore Inc.). The signals acquired by Chem documentation unit (Bio-Rad Laboratories) for various targets were normalized using $\beta$-actin as the loading control.

Statistical analysis. Statistical analysis was performed using the SPSS 10 package. Values represent the means \pm SD for 6 rats in each group, and the significance of difference between mean values was determined by one-way analysis of variance (ANOVA) followed by Tukey's multiple comparison test. 


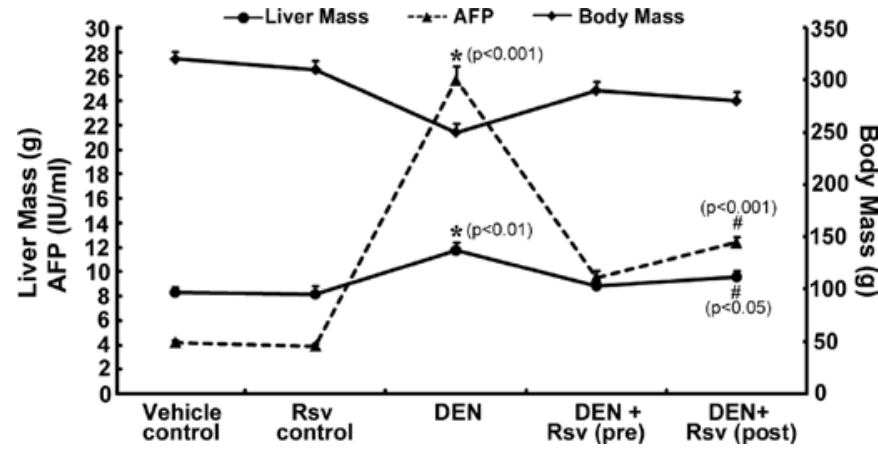

Figure 2. Effect of resveratrol (Rsv) on the serum $\alpha$-fetoprotein (AFP) level, liver mass and total body mass in DEN-induced HCC of male Wistar rats. HCC was induced by DEN and phenobarbital administration, as described in Materials and methods. DEN-treated rats showed the principal characteristics of HCC, such as increase in the level of serum AFP $(\mathrm{p}<0.001)$ and also an increase in the liver mass $(\mathrm{p}<0.01)$ with a corresponding decrease in the total body mass. Rsv-treated HCC-bearing rats showed a significant decrease in the AFP level $(\mathrm{p}<0.001)$ and liver mass $(\mathrm{p}<0.05)$ in comparison to untreated HCC-bearing rats (DEN). Total body mass also increased in HCC rats treated with Rsv. Vehicle control, rats treated with carboxymethyl cellulose; Rsv control, rats treated with Rsv; DEN, induction of HCC by DEN; DEN + Rsv (pre), DEN-induced HCC bearing rats treated with Rsv simultaneously from the 1st to 2 nd week (15 days) DEN + Rsv (post), DEN administration followed by Rsv treatment during weeks 17 and 18 (15 days). Data represent the means \pm SEM of 6 animals in each group. " p-value indicates comparison between 'Vehicle control' rats and 'DEN' and " $\mathrm{p}$-value indicates comparison between 'DEN' and 'DEN + Rsv (post)'.

\section{Results}

Effect of resveratrol on total body mass, liver enlargement and AFP, and histopathology. DEN-induced hepatocarcinogenesis in rats demonstrated the characteristics of HCC, such as an increase in the serum level of AFP, a decrease in the total body mass and liver enlargement. A comparison of 'control' and 'DEN'-administered rats showed a significant variation in these three characteristics $(\mathrm{p}<0.001)$ (Fig. 2). A statistically significant decrease in the serum level of AFP $(\mathrm{p}<0.001)$ and total liver mass $(\mathrm{p}<0.05)$ in resveratrol-treated $\mathrm{HCC}$ indicated the biochemical alterations at the cellular level in a resveratrol-dependent manner. A gain in the body mass of resveratrol-treated rats [both pre (early) and post (advanced)$\mathrm{HCC}]$ implies the antitumorigenic properties of resveratrol. Reasonably, the effect of resveratrol was more pronounced in the early- than the advanced-stage treatment, based on the statistically significant reduction of the AFP level.

Histopathological observations of liver sections from DEN-induced HCC showed crowding of cells, alteration in the hepatocyte architecture with a loss of radiating hepatocytes and sinusoidal infiltration (Fig. 3). Resveratrol-treated groups (early and advanced stages) showed a marked recovery in both hepatocyte architecture and a reduction of sinusoidal infiltration and radiating hepatocytes.

Effect of resveratrol on serum and liver marker enzymes. Elevated levels of serum marker enzymes are common during HCC pathogenesis. Herein, DEN-induced HCC also demonstrated a marked increase in the activities of several serum marker enzymes, such as aminotransferases (ALT, AST), phosphatases (ALP, ACP), 5'ND, $\gamma$-GT and LDH (Fig. 4). All these marker enzymes showed a statistically significant

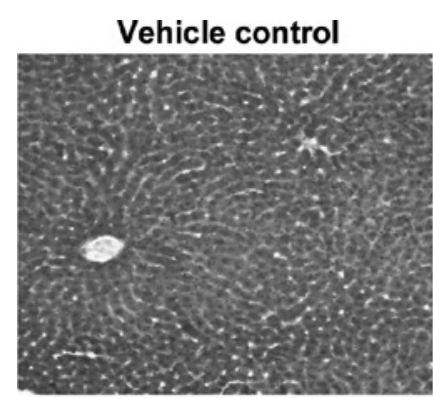

DEN + Rsv (pre)

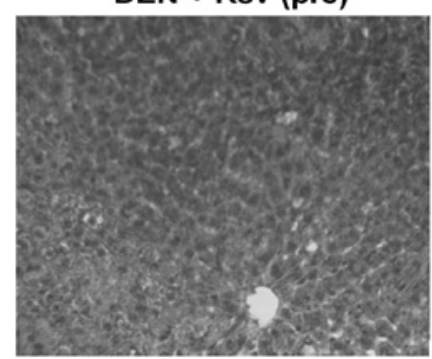

Rsv control

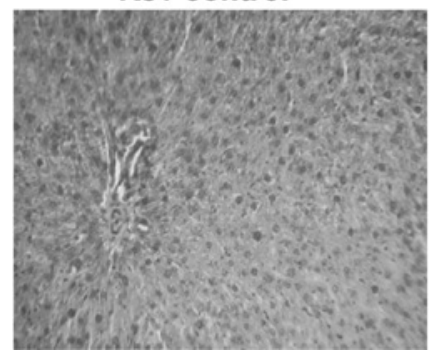

Figure 3. Effect of resveratrol on DEN-induced HCC. H\&E-stained histological sections from rat liver treated with either the controls (vehicle or Rsv), or DEN, indicating DEN-dependent hepatic histological alteration (magnification, $\mathrm{x} 40$ ). Rsv treatment in both 'DEN + Rsv (pre)' and 'DEN + Rsv (post)' affected HCC and stopped the DEN-induced alterations. Rsv, resveratrol.

increase upon $\mathrm{HCC}$ induction $(\mathrm{p}<0.001)$. Both in the pre-HCC (early) and post-HCC (advanced) treatment groups, resveratrol significantly reduced the activity of these marker enzymes compared to the untreated HCC-bearing (DEN) rats. Statistical analyses also showed that despite a significant difference in the activities of these enzymes between the resveratrol-treated and untreated groups, the level of significance varied among them. The $\mathrm{p}$-values for these enzymes were: AST, $\mathrm{p}<0.001$; ALT, $\mathrm{p}<0.001 ;$ ALP, $\mathrm{p}<0.05$; ACP, $\mathrm{p}<0.01 ; 5$ '-Nuc, $\mathrm{p}<0.01$; $\gamma-\mathrm{GT}, \mathrm{p}<0.001$; and $\mathrm{LDH}, \mathrm{p}<0.001$. It is of note that the resveratrol-treated 'control' rats (without HCC) did not show any alteration in their enzyme activities, reiterating that the resveratrol-dependent effect on enzyme activities is selective or limited to HCC-bearing rats.

Analysis of liver marker enzymes showed that DEN-induced HCC significantly increased the activities of the enzymes, such as AST $(p<0.01)$, ALT $(p<0.001)$, ALP $(\mathrm{p}<0.05)$, ACP $(\mathrm{p}<0.001), 5$ '-Nuc $(\mathrm{p}<0.05), \gamma-\mathrm{GT}(\mathrm{p}<0.001)$ and LDH $(\mathrm{p}<0.001)$ (Fig. 5). Similar to serum marker enzymes, the activity of liver marker enzymes also showed a decrease in their activities in a resveratrol treatment-dependent fashion in HCC-bearing rats. Notably, there was a statistically significant variation between the early and advanced HCC treatment groups of resveratrol, where pre-treated rats showed a marked decrease in the activity compared to the post-HCC treated rats. 


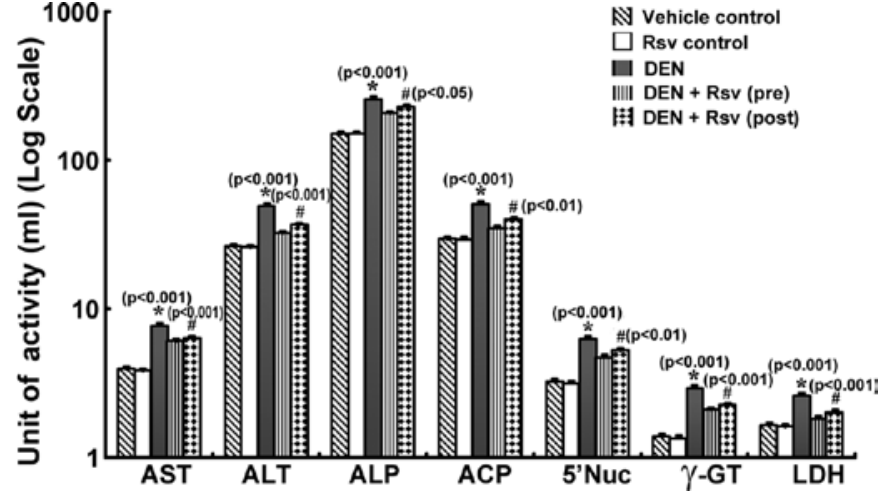

Figure 4. Effect of resveratrol (Rsv) on the activity of serum marker enzymes in DEN-induced HCC rats. Major serum marker enzymes were assayed for their activity as described in Materials and methods. Data show that the activity of serum marker enzymes increased significantly $(\mathrm{p}<0.001)$ in DENinduced HCC. Rsv treatment at pre [DEN + Rsv (pre)] and post [DEN + Rsv (post)] HCC induction significantly reduced the activity of serum marker enzymes close to the 'vehicle control'. Vehicle control, rats treated with carboxymethyl cellulose; Rsv control, rats treated with Rsv; DEN, refers to induction of HCC by DEN; DEN + Rsv (pre), DEN-induced HCC-bearing rats treated with Rsv simultaneously from the 1st to 2nd week (15 days); DEN + Rsv (post), DEN administration followed by Rsv treatment during weeks 17 and 18 (15 days). Data represent the means \pm SEM of 6 animals in each group. " $\mathrm{p}$-value indicates comparison between the 'vehicle control' rats and 'DEN' and ${ }^{\#} \mathrm{p}$-value indicates comparison between 'DEN' and 'DEN + Rsv (post)'.

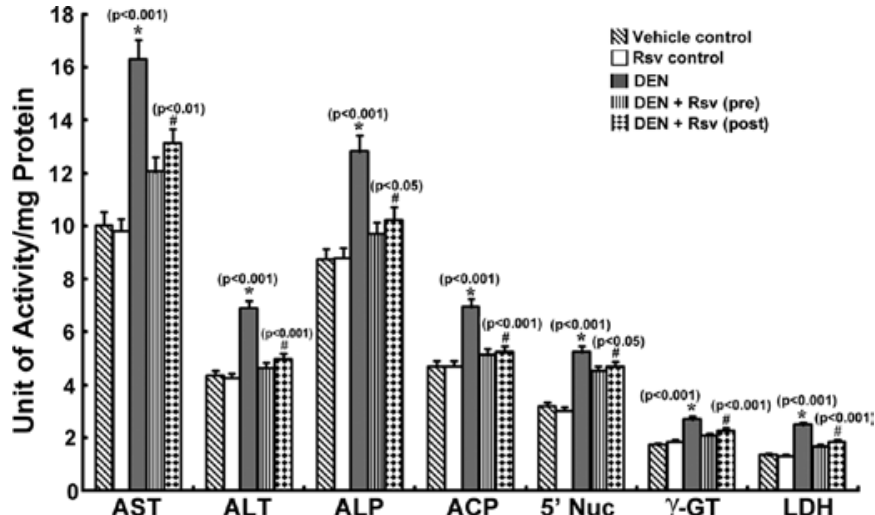

Figure 5. Effect of resveratrol (Rsv) on the activity of liver marker enzymes in DEN-induced HCC rats. Liver marker enzymes showed a significant $(p<0.001)$ increase in their level of activity upon induction of HCC by DEN Rsv treatment at pre [DEN + Rsv (pre)] and post [DEN + Rsv (post)] HCC induction significantly reduced the activity liver marker enzymes close to the 'vehicle control'. Vehicle control, rats treated with carboxymethyl cellulose; Rsv control, rats treated with Rsv; DEN, induction of HCC by DEN; DEN + Rsv (pre), DEN-induced HCC-bearing rats treated with Rsv simultaneously from the 1st to 2nd week (15 days); DEN + Rsv (post), DEN administration followed by Rsv treatment during weeks 17 and 18 (15 days). Data represent the means \pm SEM of 6 animals in each group. "p-value indicates comparison between 'vehicle control' rats and 'DEN'and " $\mathrm{p}$-value indicates comparison between 'DEN' and 'DEN + Rsv (post)'.

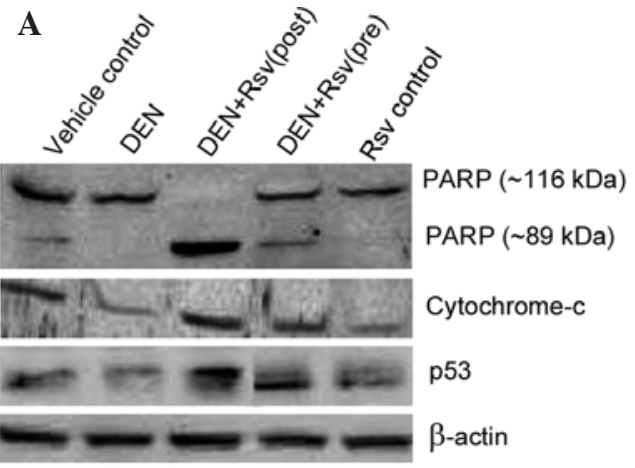

B
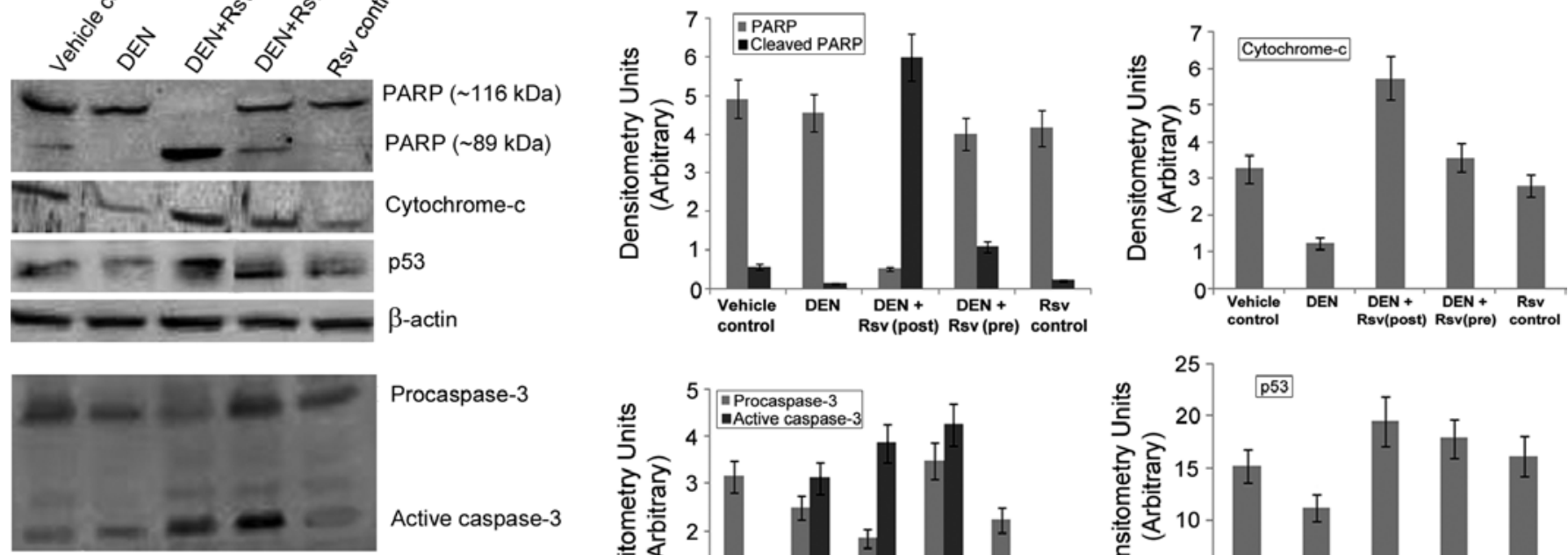

Procaspase-3
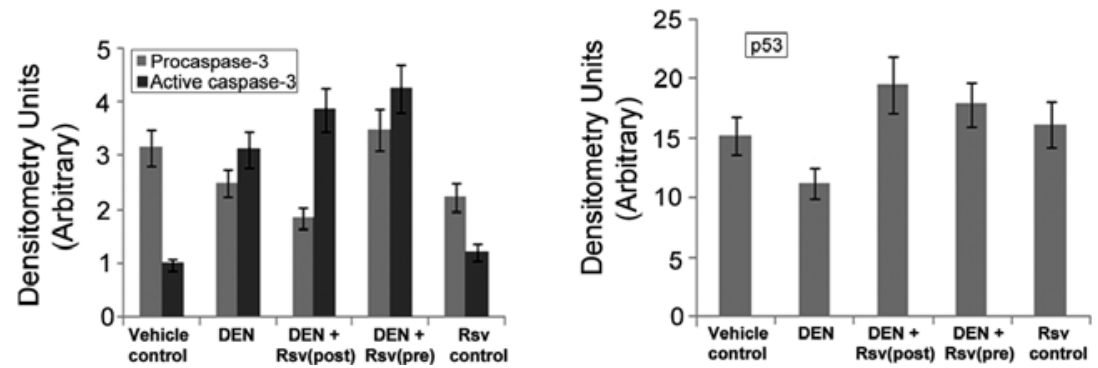

Figure 6. Resveratrol (Rsv) treatment induced apoptosis in rat HCC. (A) Immunoblot showing the apoptotic events, such as cleavage of PARP, release of cytochrome-c, p53 induction and the activation of procaspase-3 into caspase-3 in Rsv-treated HCC. The blot is representative of three independent experiments. $\beta$-actin was used as the loading control. (B) Representation of densitometry values normalized with $\beta$-actin for the signal intensities of respective targets in immunoblots. DEN + Rsv (pre), treatment with Rsv prior to HCC induction; DEN + Rsv (post), treatment with Rsv post HCC formation.

Effect of resveratrol on apoptotic regulators. Immunoblots showed that the resveratrol treatment promoted PARP cleavage, cytochrome-c release, induction of p53 and conversion of procaspase-3 into active caspase-3 in HCC rats (Fig. 6), indicating a resveratrol-dependent induction of the apoptotic pathway. PARP cleavage or conversion was very profound in the 'DEN + Rsv (post)' induction group compared to the 'DEN + Rsv (pre)' group. The level of cytochrome-c also demonstrated a similar trend. Intriguingly, for unknown reasons, a significant amount of cytochrome-c release was observed even in the 'control' liver. It has yet to be elucidated whether mitochondrial stress in any of these 'control' rats is responsible for this.

The expression of $\mathrm{p} 53$ increased in a resveratrol treatmentdependent manner demonstrating the activation of the p53-related apoptotic pathway (Fig. 6). A significant decrease in p53 expression in HCC-bearing rats substantiates the anti-apop- 
A

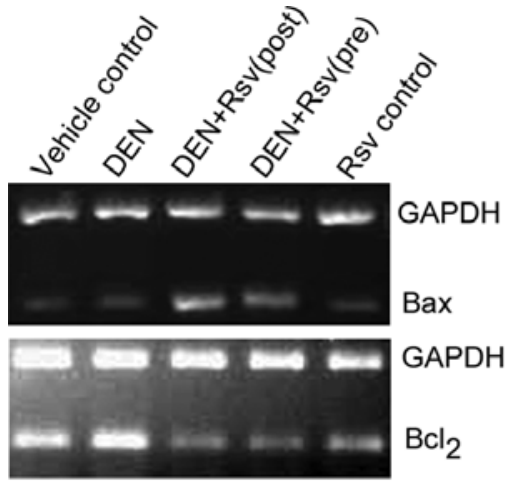

C

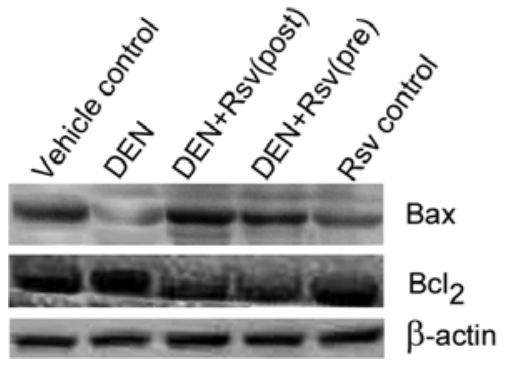

B
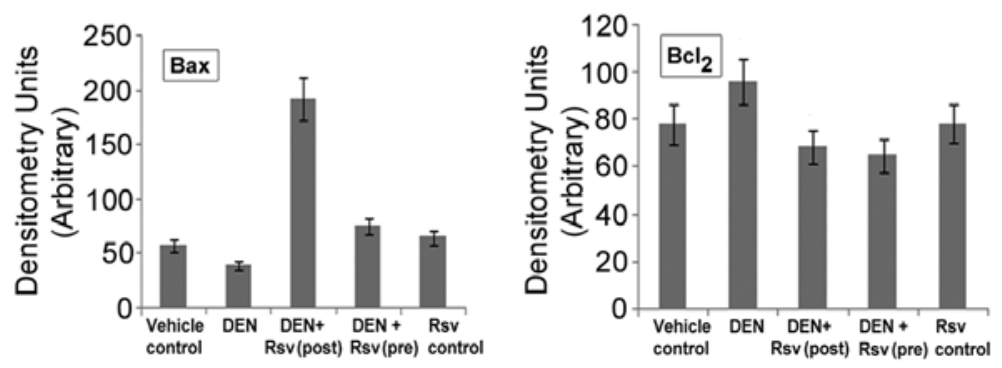

D

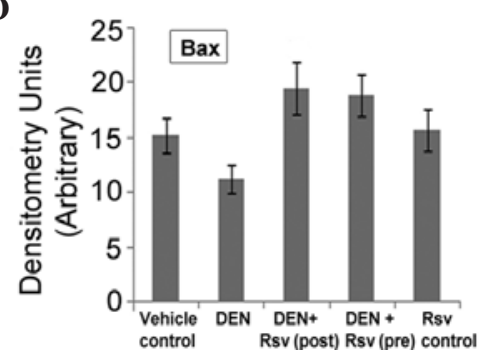

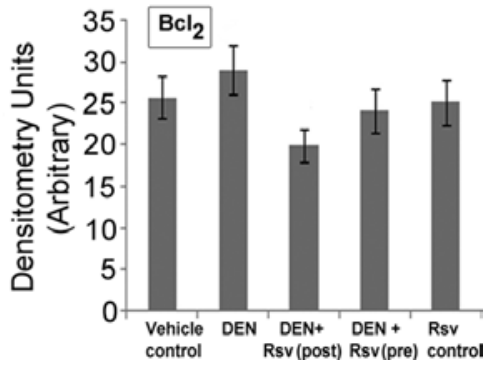

Figure 7. Resveratrol (Rsv) treatment inhibited $\mathrm{Bcl}_{2}$ and induced Bax in rat HCC. (A) Semi-quantitative RT-PCR and (B) the corresponding densitometry values normalized with GAPDH. (C) Immunoblots showing the down-regulation of $\mathrm{Bcl}_{2}$ and up-regulation of Bax in a Rsv treatment-dependent manner. (D) Corresponding densitometry values normalized with $\beta$-actin. DEN, N-nitrosodiethylamine; DEN + Rsv (pre), treatment with Rsv prior to HCC induction; DEN + Rsv (post), treatment with Rsv post HCC formation.

totic and pro-oncogenic mechanism involved in DEN-induced hepatocarcinogenesis in rats. Resveratrol treatment at both the early and advanced stages of DEN-induced HCC increased the expression level of $\mathrm{p} 53$, supporting the activation of a common apoptotic mechanism during the preventive and therapeutic effects.

Notably, the resveratrol-mediated activation of caspase-3 was not seen in the 'control' rats without HCC, but only in the HCC-bearing rats treated with resveratrol. This suggests that the activation of the caspase- 3 by resveratrol treatment is specific to HCC cells, but not healthy hepatocytes or liver parenchyma. The therapeutic effect of resveratrol is more evident when used in the early than in the advanced stages of HCC. However, in terms of the rate of conversion of procaspase-3 into active caspase-3, a higher rate of conversion was observed in the advanced-stage treatment group, implying an increased demand/requirement for the induction of apoptosis.

Similarly, the resveratrol treatment affected the expression of apoptotic regulators, such as $\mathrm{Bcl}_{2}$ (anti-apoptotic) and Bax (pro-apoptotic), at both the transcriptional (mRNA) and translational (protein) levels in HCC-bearing rats. Semiquantitative PCR analysis showed an induction of the $\mathrm{Bcl}_{2}$ mRNA level during the DEN-induced hepatocarcinogenesis, whereas the resveratrol treatment mitigated its expression, blocking its anti-apoptotic role (Fig. 7). However, the level of the pro-apoptotic protein, Bax, was markedly increased in resveratrol-treated $\mathrm{HCC}$, at both mRNA and protein levels, favoring the apoptotic signaling pathway (Fig. 7).

\section{Discussion}

A number of epidemiological studies have indicated that a diet rich in fruits and vegetables is associated with the reduc- tion of cancer risk in humans, suggesting that certain dietary constituents may thus be effective in preventing cancer (17). Most cancer preventive agents are natural polyphenols which act by preventing enzymes involved in carcinogen activation (phase I). Polyphenolic compounds constitute a diverse group of secondary metabolites that are present in the human diet. This group of heterogeneous compounds corresponds to a wide variety of chemopreventive agents and has been categorized in the following groups: Phenolic acids, stilbenes, flavonoids and analogs. Many of the individual phytochemicals are primarily potent antioxidants with the potential to arrest tumorigenesis. These natural agents generally down-regulate signaling pathways which have been activated in malignant cells, and block the proliferation of initiated cells with minimal damage to normal cells.

Many studies have highlighted the chemopreventive potential of resveratrol in experimental hepatocarcinogenesis in rodent models $(12,18,19)$. Bishayee and Dhir (12) demonstrated the chemopreventive effects of resveratrol in DEN-induced hepatocarcinogenesis in female Sprague Dawley rats. However, to date, there is no report on the effects of resveratrol during the early and advanced stages of DEN-induced hepatocarcinogenesis. DEN induction of HCC has long been known and has been extensively characterized for liver cancer-related biology. Hence, we attempted to study the effects of resveratrol on DEN-induced HCC at the early and advanced stages. Also, as a great amount of epidemiological data have shown that HCC is more prevalent in men than women $(1,20)$, the evaluation of the therapeutic effects of resveratrol in male animal models is critical and clinically relevant. Hence, in the present study, we document the effects of early- and advanced-stage intervention with resveratrol on DEN-induced HCC in male Wistar rats. Conventional studies on the chemopreventive effects of resve- 
ratrol involve the administration of resveratrol several weeks prior to the induction of HCC. To the best of our knowledge, there is no report on the effects of resveratrol during concurrent administration of both the HCC-inducer (DEN) and resveratrol. Thus, the intervention by resveratrol in DEN-induced HCC is addressed in our study, and the effects of resveratrol on the early and advanced stages of DEN-induced HCC are compared.

Since the report of Jang et al (7) on the chemopreventive potential of resveratrol, many studies have supported its anticancer effects. It is now well established that resveratrol has anticancer effects, both in terms of prevention and therapy, in various tumor types irrespective of their origin or phenotype (5). Multiple lines of evidence have indicated that HCC cells of human or rodent origin are sensitive to resveratrol treatment. For example, in in vitro experiments the rat hepatoma cell line, Fao, and the HepG2, human HCC cell line have been found to be more sensitive to resveratrol treatment in a dose- and time-dependent manner (21). It is ascribed that cell-cycle arrest or a delay in mitosis are probable mechanism(s) underlying resveratrol's antiproliferative effects. This hypothesis has gained support based on the data from other reports as well $(22,23)$. However, it was Sun et al (24) who provided the direct evidence that resveratrol up-regulated the apoptotic signaling pathway during its cytotoxic effects in H22 hepatoma cells. Furthermore, support for the involvement of the apoptotic pathway as the primary cytotoxic mechanism underlying resveratrol's anticancer effect was demonstrated in H4IIE cells (25). The activation of the p53 pathway upon resveratrol treatment as observed in the present study is in agreement with an earlier report (26). The present study demonstrates the up-regulation of Bax and the down-regulation of $\mathrm{Bcl}_{2}$, the two key pro-apoptotic and anti-apoptotic proteins, respectively, in rat HCC.

It is estimated that, on average, almost 500,000 new cases of HCC are identified every year. The high mortality rate is also due to the short survival period of patients (5-year fatality rate is over 95\%) after diagnosis. Although the prevalence of HCC is high in Africa and Asia, its incidence in Western countries is also rising, mainly due to the increasing rates of alcoholic liver disease and hepatitis $\mathrm{C}$ infection. HCC is considerably more common in men than women, with an incidence ratio of approximately 3:1 (2). HCC is resistant to systemic chemotherapies, hence a cancer-specific agent with minimal or no toxicity to normal, healthy cells is the current requirement to fight against cancer. Our results demonstrate that resveratrol-dependent effects are selective or preferential to HCC-bearing rats with no or minimal effects on control (non-HCC) rats. This indicates that resveratrol has the desirable properties of an anticancer agent and has the translational potential for clinical investigation.

\section{Acknowledgements}

The financial support, in the form of Senior Research Fellowship from CSIR, Government of India to Devaraja Rajasekaran (CSIR Sanction No. 9/115(0686)/2008-EMR-I), is gratefully acknowledged.

\section{References}

1. Minguez B, Tovar V, Chiang D, Villanueva A and Llovet JM: Pathogenesis of hepatocellular carcinoma and molecular therapies. Curr Opin Gastroenterol 25: 186-194, 2009.
2. Bosch FX, Ribes J, Cleries R and Diaz M: Epidemiology of hepatocellular carcinoma. Clin Liver Dis 9: 191-211, 2005.

3. Bosch FX, Ribes J and Borras J: Epidemiology of primary liver cancer. Semin Liver Dis 19: 271-285, 1999.

4. Llovet JM, Ricci S, Mazzaferro V, et al: Sorafenib in advanced hepatocellular carcinoma. N Engl J Med 359: 378-390, 2008.

5. Cal C, Garban H, Jazirehi A, Yeh C, Mizutani Y and Bonavida B: Resveratrol and cancer: chemoprevention, apoptosis, and chemoimmunosensitizing activities. Curr Med Chem Anticancer Agents 3: 77-93, 2003 .

6. Bishayee A, Politis T and Darvesh AS: Resveratrol in the chemoprevention and treatment of hepatocellular carcinoma. Cancer Treat Rev 36: 43-53, 2010.

7. Jang M, Cai L, Udeani GO, et al: Cancer chemopreventive activity of resveratrol, a natural product derived from grapes. Science 275: 218-220, 1997.

8. Bishayee A, Darvesh AS, Politis T and McGory R: Resveratrol and liver disease: From bench to bedside and community. Liver Int 30: 1103-1114, 2010.

9. Bishayee A: Cancer prevention and treatment with resveratrol: from rodent studies to clinical trials. Cancer Prev Res 2: 409-418, 2009.

10. Aggarwal BB, Bhardwaj A, Aggarwal RS, Seeram NP, Shishodia S and Takada Y: Role of resveratrol in prevention and therapy of cancer: preclinical and clinical studies. Anticancer Res 24: 2783-2840, 2004.

11. Kweon S, Kim Y and Choi H: Grape extracts suppress the formation of preneoplastic foci and activity of fatty acid synthase in rat liver. Exp Mol Med 35: 371-378, 2003.

12. Bishayee A and Dhir N: Resveratrol-mediated chemoprevention of diethylnitrosamine-initiated hepatocarcinogenesis: inhibition of cell proliferation and induction of apoptosis. Chem Biol Interact 179: 131-144, 2009.

13. King J: Practical clinical enzymology. Van Nostrand, London, New York, p363, 1965.

14. Orlowski M and Meister A: Isolation of gamma-glutamyl transpeptidase from hog kidney. J Biol Chem 240: 338-347, 1965.

15. Lowry OH, Rosebrough NJ, Farr AL and Randall RJ: Protein measurement with the folin phenol reagent. J Biol Chem 193: 265-275, 1951.

16. Laemmli UK: Cleavage of structural proteins during the assembly of the head of bacteriophage T4. Nature 227: 680-685, 1970.

17. Guo W, Kong E and Meydani M: Dietary polyphenols, inflammation, and cancer. Nutr Cancer 61: 807-810, 2009.

18. Carbo N, Costelli P, Baccino FM, Lopez-Soriano FJ and Argiles JM: Resveratrol, a natural product present in wine, decreases tumour growth in a rat tumour model. Biochem Biophys Res Commun 254: 739-743, 1999.

19. Luther DJ, Ohanyan V, Shamhart PE, et al: Chemopreventive doses of resveratrol do not produce cardiotoxicity in a rodent model of hepatocellular carcinoma. Invest New Drugs 29: 380-391, 2011.

20. Sloane D, Chen H and Howell C: Racial disparity in primary hepatocellular carcinoma: tumor stage at presentation, surgical treatment and survival. J Natl Med Assoc 98: 1934-1939, 2006.

21. Delmas D, Jannin B, Cherkaoui Malki M and Latruffe N: Inhibitory effect of resveratrol on the proliferation of human and rat hepatic derived cell lines. Oncol Rep 7: 847-852, 2000.

22. Hsieh TC, Burfeind P, Laud K, Backer JM, Traganos F, Darzynkiewicz Z and Wu JM: Cell cycle effects and control of gene expression by resveratrol in human breast carcinoma cell lines with different metastatic potentials. Int J Oncol 15: 245-252, 1999.

23. Notas G, Nifli AP, Kampa M, Vercauteren J, Kouroumalis E and Castanas E: Resveratrol exerts its antiproliferative effect on HepG2 hepatocellular carcinoma cells, by inducing cell cycle arrest, and NOS activation. Biochim Biophys Acta 1760: 1657-1666, 2006

24. Sun ZJ, Pan CE, Liu HS and Wang GJ: Anti-hepatoma activity of resveratrol in vitro. World J Gastroenterol 8: 79-81, 2002.

25. Michels G, Watjen W, Weber N, et al: Resveratrol induces apoptotic cell death in rat H4IIE hepatoma cells but necrosis in C6 glioma cells. Toxicology 225: 173-182, 2006.

26. Kuo PL, Chiang LC and Lin CC: Resveratrol-induced apoptosis is mediated by p53-dependent pathway in hep G2 cells. Life Sci 72: 23-34, 2002. 